\title{
Correspondence
}

\section{Levamisole and surgery in bronchial carcinoma patients: increase in deaths from cardiorespiratory failure}

Sir,-We would like to comment on this paper by Dr Anthony and her coworkers.

Firstly, whether or not these deaths are related to levamisole must be examined in relation to three factors:

1. (a) In a three-centre study (which inspired Anthony et al to start their own trial) of 211 resectable lung cancer patients the percentage of non-tumour deaths after three months (the period during which the excess death rate in the Leeds study was reported) was three with levamisole $(n=96)$ and four with the placebo $(n=115)$. In view of the findings in Leeds and at the request of Dr Anthony we asked each centre (Amsterdam, Leuven, and Utrecht) to review all early deaths, particularly those diagnosed as myocardial infarction or bronchopneumonia. We also requested a report on any surviving patients who had shown periods of unexplained breathlessness and/or a pulmonary oedemalike syndrome that failed to respond to diuretics. No centre was able to find a single suspected case in the levamisole group.

(b) A similar request was sent to Dr P Wright (Fred Hutchinson Cancer Center, Seattle) who is involved in another double-blind resectable lung cancer study with levamisole started preoperatively (as in Leeds and in the study 1(a) above). Dr Wright reports that an interim analysis of his trial shows no excess deaths of a similar nature to those reported by $\mathrm{Dr}$ Anthony. This is important in view of Dr Anthony's comment that the increased death rate was pronounced in patients weighing more than $75 \mathrm{~kg}$ and that these heavy patients were treated with $50 \mathrm{mg}$ four times a day-all other patients received $50 \mathrm{mg}$ three times a day. In Seattle all patients weighing more than $70 \mathrm{~kg}$ are treated with at least $4 \times 50 \mathrm{mg}$ daily.

2. From the original data that $\mathrm{Dr}$ Anthony showed us which covered non-tumour deaths as a proportion of all operations (including exploratory thoracotomy) month by month we have derived the following table in which the data are split into four periods:

\begin{tabular}{lllr}
\hline Period of operation & \multicolumn{2}{l}{ Non-tumour deaths with: } \\
\cline { 3 - 4 } & & Levamisole & \multicolumn{1}{c}{ Placebo } \\
\hline I & Oct $75-$ Apr 76 & $31 \%(9 / 29)$ & $3 \%(1 / 39)$ \\
II & May 76-Oct 76 & $19 \%(9 / 47)$ & $9 \%(3 / 35)$ \\
III & Nov 76-Apr 77 & $17 \%(8 / 47)$ & $7 \%(3 / 45)$ \\
IV & May 77-Oct 77 & $7 \%(2 / 30)$ & $11 \%(3 / 28)$ \\
\hline
\end{tabular}

From this it seems that the non-tumour deaths in the levamisole group tend to diminish strikingly with time. The difference between period I and the other three periods approaches statistical significance $(P=$ $0 \cdot 06$, Fisher exact probability test, two-tailed). This is not the case in the placebo group $(P=0.29)$ where there is a reverse trend. In period IV the incidence of nontumour deaths in the levamisole group is below the placebo incidence for the same period and the levamisole incidence is highest when the placebo incidence is lowest (period I).

What is not shown is the incidence per centre: from individual data that Dr Anthony showed us we gained the impression that the major excess in death rates was in the first six months of the study in each of the two treatment centres where excess mortality was observed. It would be useful if this aspect were further clarified.

3. The possibility that an abnormally low incidence of non-tumour deaths in the placebo group could have contributed to their observation was only briefly mentioned in the Leeds paper. Nevertheless, they reported an unusually high survival rate in the placebo patients with blood group $O$ and there was no likely explanation available for the latter phenomenon.

Secondly, the proposed mechanism (autoimmune heart disease) to explain the deaths appears extremely speculative as:

1. This is based on the finding of antiheart antibodies in the serum of only three levamisole patients who developed signs of cardiorespiratory failure;

2. Even if all these patients had such antibodies, this could not be considered conclusive evidence since it is well known that this type of antibody (ie directed against cell constituents, not against the membrane) is usually the consequence of tissue damage, not the cause of it. One should also have similar data on placebo patients who died from a similar syndrome;

3. Autoimmune antibodies have repeatedly been evaluated in patients treated with levamisole: there is no evidence that this treatment may induce the occurrence of such antibodies. On the contrary, it has been found in several studies that such antibodies, if present before the start of treatment, tend to disappear after prolonged treatment with the drug.

In conclusion, we consider that there are two likely interpretations of the findings reported by Anthony et al:

1. An unknown factor, with an occurrence that was limited in time (ie the early trial period), has interfered with the trial in Leeds but nowhere else up to now. This exogenous factor, whose identity is still an enigma, might have adversely interacted with the levamisole treatment around the time of surgery in particularly susceptible patients.

2. The observations in Leeds were coincidental and would have tended to disappear on the entry of more patients into the trial (as suggested by the findings in 
the last six monhts of the study). The confirmation or rejection of this interpretation will never be possible since the trial has, understandably, been prematurely stopped. Nevertheless, the fact is that we should not forget that exceptional events, however unlikely, do occur in exceptional circumstances.

Only future observations from other continuing studies will tell us whether the findings in Leeds are unique or not.

WILLEM K AMERY, MD Janssen Pharmaceutica NV, Beerse, Belgium.

REPLY - I am glad you have given Dr Amery a chance to state the position with regard to non-cancer deaths in other trials and me a chance to reply.

As Dr Amery says, there was no significant excess of non-cancer deaths in his trial or in that of $\mathrm{Dr}$ Wright in Seattle. I understand, in addition, that neither of them is convinced that any of the deaths that did occur showed similarity to ours.

Three things about this are important. Firstly, that the original analysis in each case omitted non-cancer deaths, considering only cancer deaths (Amery, 1976) or cancer recurrences (Wright et al, 1977). This is not uncommon in trial analysis although the points against have been well made (Peto et al, 1976, 1977) and are underlined by our experience. Secondly, they have been good enough to go over their cases since they heard our story and have each found marginally more non-cancer deaths on levamisole than on placebo. Thirdly, so far as I know, the case notes of these patients have not been submitted blind to an uninterested party. What I have seen of their data has not convinced me that it would be safe to conclude that their experience is qualitatively at variance with ours, although there can be no doubt that the size of the problem is quite different.

I am doubtful if the excess deaths in our trial were different in mechanism from occasional deaths after lung resection without the drug. It may be that levamisole regularly potentiates an event that is normally rare and that some other agent interacted with levamisole in our study to push this up to levels where it caused a significant difference in survival. For this reason, detection of antiheart antibody in the serum (had we had it) of any of the few placebo-treated patients dying in respiratory distress would not have disproved our hypothesised explanation and might have contributed to our understanding of the hazards of lung resection.

I was hampered in suggesting that another agent had interacted with levamisole in causing the effect by the fact that $I$ could find no evidence for it apart from the differences between our trial and the others. Dr Amery points to an apparent decrease in excess deaths over the period of the trial and asks for a breakdown by centre. This does not support his contention. The deaths were randomly distributed throughout the period in one centre. In the other centre the same was true apart from a two-month period early in the trial with $4 / 7$ deaths in levamisole and $2 / 7$ in placebotreated resected patients. The overall excess was comparable for the two centres so that one would be un- $\stackrel{\mathbb{Q}}{\Omega}$ wise to attribute the pattern to anything other than chance.

In Dr Amery's reading of the literature, levamisole $\overrightarrow{0}$ does not increase and may decrease autoantibody production. However, we are not the first to attribute $\omega_{\mathscr{O}}$ side effects of levamisole to autoantibody, and in view of the complexity of immunological mechanisms it $\vec{x}$ would be simplistic to conclude that levamisole never $\omega$ increases autoantibody production when it has been shown to increase macrophage phagocytosis and pinocytosis and may therefore affect antigen presentation $\triangle$ to $B$ and $T$ cells. We do not know what controls 0 autoimmune disease, but we do know that genetic predisposition is involved since most of these diseases are commoner in individuals of group $\mathrm{O}$ and/or certain HLA types (Mourant et al, 1978).

Contrary to the implication of Dr Amery's letter, our trial entry already exceeded the planned accrual when the trial was stopped six weeks early on finding the excess deaths. Except for the patients who had just $v$ started the drug (from whom it was withdrawn) patients are continuing as planned even though any beneficial effect of levamisole will be very difficult to establish after such a disastrous start.

Like Dr Amery, we should not like this drug to be discarded on inadequate grounds, but we would beg other workers to ensure that all postoperative deaths are included in trials analyses and that repeated serum samples are obtained from all patients treated with levamisole preoperatively so that changes in titre of both viral and autoantibodies can be looked for in all cases dying in hospital.

HONOR M ANTHONY

\section{References}

Amery, W K (1976). Double-blind levamisole trial in resectable lung cancer. Annals of the New York Academy of Sciences, 277, 260-268.

Mourant, A E, Kopec, A C, and Domaniewska- $\frac{\bigcirc}{9}$ Sobczak, K (1978). Blood Groups and Diseases, p 49. Oxford University Press, London.

Peto, R, Pike, M C, Armitage, P, Breslow, N E, Cox, D R, Howard, S V, Mantel, N, McPherson, K, Peto, N J, and Smith, P G (1976)* (1977) †. Design and analysis of randomised clinical trials requiring prolonged observation of each patient.

Wright, P W, Hill, L D, Anderson, R P, Hammar, S P, Bernstein, I D, and Prentice, R L (1977). In Adjuvant Therapy of Cancer, edited by $\mathrm{S}$ E Salmon and 0 $\mathrm{S}$ E Jones, pp 217-224. Élsevier, Amsterdam.

*Introduction and design. British Journal of Cancer,

34, 585-612.
+ Analysis and examples. British Journal of Cancer, 35, 1-39. 